\title{
Telesonar Testbed Instrument Provides a Flexible Platform for Acoustic Propagation and Communication Research in the $8-50 \mathrm{kHz}$ Band
}

\author{
Vincent K. McDonald ${ }^{*}$, Paul Hursky ${ }^{\dagger}$, and the KauaiEx Group \\ * Space and Naval Warfare Systems Center, San Diego, CA. 92152 \\ ${ }^{\dagger}$ Center for Ocean Research, SAIC, 10260 Campus Point Drive, San Diego CA, 92121
}

\begin{abstract}
The underwater channel remains a difficult medium for transmitting communication signals. Frequent field tests are required for validating models, testing new waveforms and coding schemes, developing link protocols, designing an adaptive multi-mode modem, testing third-party prototype modems, testing a new directional transducer, and developing new DSPefficient algorithms. A modular, flexible, autonomous instrument was designed to easily and inexpensively conduct such field tests. This instrument, called the Telesonar Testbed, was originally designed five years ago to specifically support the Telesonar Program at the Space and Naval Warfare Systems Center, San Diego. It has since taken on a wider role supporting the High-Frequency Initiative, the SignalEx project, and a new project testing Multiple Input/Multiple Output (MIMO) systems. Over the last five years several major design changes have been made, making it smaller, lighter weight, more reliable, and acoustically commandable. This paper will describe the design and features of this instrument which has been the centerpiece of 10 experiments to date. This paper will also apply six carrier estimation and six symbol timing estimators to data received by the Telesonar Testbeds in the KauaiEx and ElbaEx Experiments.
\end{abstract}

\section{INTRODUCTION}

The Telesonar Testbed is a unique, high-fidelity, modular, reconfigurable, autonomous, wideband instrument for high-frequency acoustic propagation and communication research. Central to its design is its ability to provide ample experimentation opportunities at low cost. Autonomous operation and a lightweight and small package allows for a meaningful experiment using a craft as small as 7 meters in length. It has been the workhorse of many experiments including the coastal waters off of California, Massachusetts, Hawaiian Island of Kauai, and most recently in the northwest Italian coastal waters near Elba Island. This paper will discuss the design and features of this instrument and present the results of applying carrier and symbol synchronization techniques to data collected by a Telesonar Testbed deployed at the KauaiEx and ElbaEx Experiment sites. 


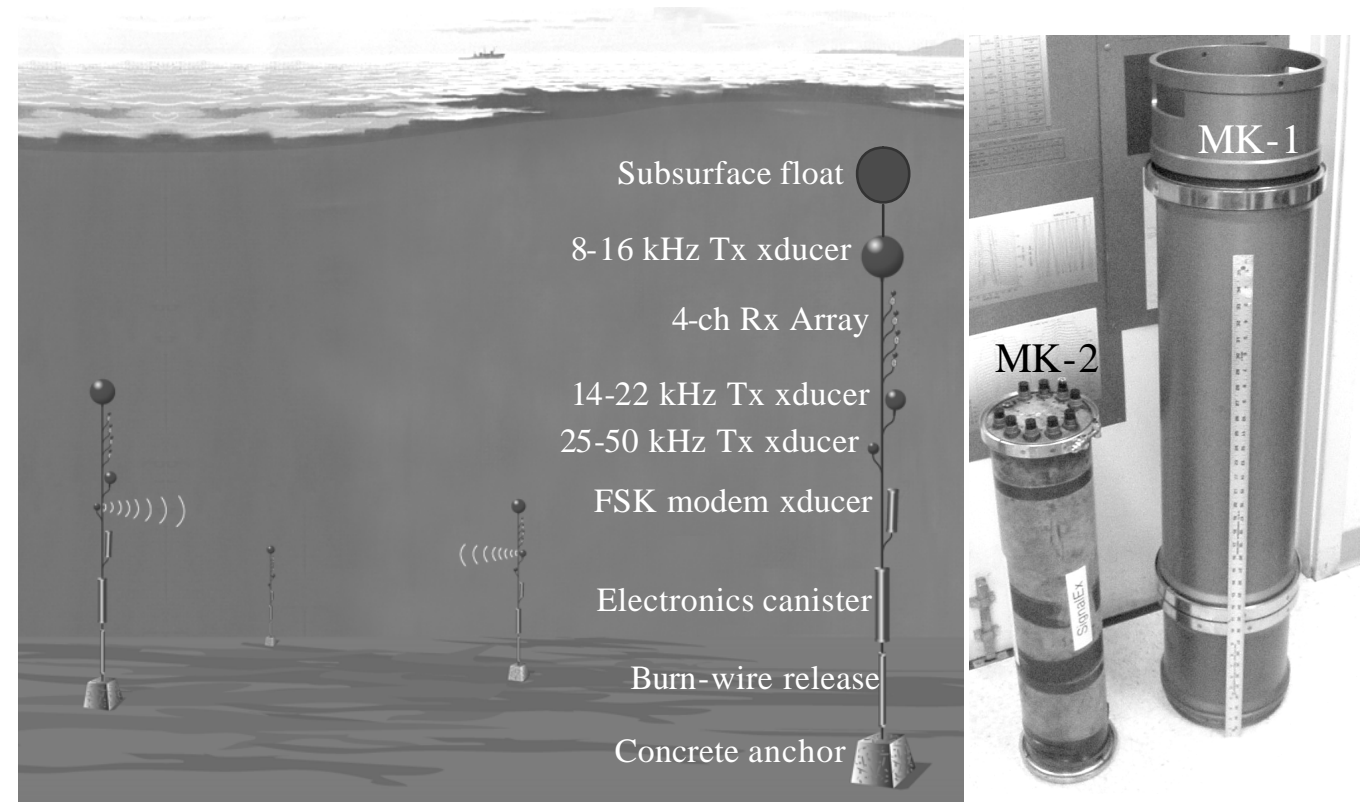

FIGURE 1. Telesonar Testbed bottom-deployed vertical configuration (left). Electronics canisters for MK-1 and MK-2 (right).

The Telesonar Testbed design and development was originally funded in 1997 by the Office of Naval Research to support the Telesonar Program in addressing underwater acoustic communication issues such as shallow-water model validation, link protocol development, multimode adaptive modem research, assessment of transmission security, spatial diversity testing, and ocean impulse response observations to name a few. Since 1997 the instrument has undergone two significant design overhauls, each time reducing the size and power consumption while increasing its capabilities. Figure 1 (right) depicts the original Testbed (MK-1), and the current instrument (MK-2).

\section{TESTBED INSTRUMENT DESCRIPTION}

\section{Instrument Mooring}

The instrument mooring is typically a serial, in-line configuration shown in Fig 1 (left). Starting from the bottom and working upwards, one to three commercially available concrete footings each with a mass of $16 \mathrm{~kg}$ in water are used to anchor the instrument to the seafloor. Above that is a pair of ultra-lightweight $(0.2 \mathrm{~kg}$ in water) releases that upon an acoustic command will "burn" a small wire through an electrically accelerated anodic dissolution process. Next is the instrument itself with internal electronics and an alkaline battery pack. If the instrument is configured as a transmitter, an additional dual-chemistry (alkaline and NiCad) transmit battery pack is strapped to the electronics canister to provide the necessary additional battery capacity and current sourcing capability. A set of transmit and receive transducers are located 
above the electronics canister. Finally, a subsurface float is used to keep transducers stable and the instrument mooring vertical in the water column in addition to providing the necessary buoyancy for instrument recovery once released from the seafloor.

The Space and Naval Warfare Systems Center, San Diego finished building four Testbed instruments in May of 2003. The instruments are configurable as a transmitter, receiver or both; however, typically one transmits while the other three, deployed at various ranges, receive and record the acoustic signals. While testing multi-access signaling methods during the KauaiEx and the ElbaEx Experiments, two instruments were configured as transmitters and two as receivers. In addition to being bottom moored, the instrument can be towed at speeds up to five knots while mounted in a custom-built tow body.

\section{Mission Description}

Before continuing with the design and features of this instrument, a mission description within the context of an experiment is in order. While the instruments are within the lab or sealed and on deck, mission parameters are loaded through a serial connection or via an RF link. The instrument is then placed in the mooring string and necessary mechanical and electrical connections made before it is lowered to the bottom or is deployed in a freefall manner. Once on the bottom or lowered to its operating depth within the tow body, a commercially-available FSK (frequency shift keying) modem enables checkout of the instrument.

At a prescribed time, the transmitter begins transmitting a probe signal followed by a communication waveform. The receiver, being cognizant of the mission plan, selects the appropriate receive array, sample rate, and opens and closes files commensurate with each short $(\leq 1 \mathrm{~min})$ transmission for ease of postmortem analysis.

During the KauaiEx Experiment, an interleaved, multi-band, transmission schedule was attempted for the first time. In an attempt at maximizing our data collection efficiency/density, the transmissions were time-division multiplexed from the towbody and the bottom mounted instrument. That way, we obtained transmissions from a fixed transmitter to a fixed receiver and a moving transmitter to a fixed receiver. Further complicating matters, transmissions would cycle through the three transmit bands, i.e. 8-16, 14-22, and 25-50 kHz. The clock accuracy required for this interleaving, required the late addition of very accurate real-time clocks in each instrument coupled with edge-triggered hardware circuitry.

\section{Mechanical Description}

The Testbed electronics and external battery canisters measure roughly $15-\mathrm{cm}$ diameter by $78-\mathrm{cm}$ length. The mass of the entire receiver mooring minus the concrete anchors (i.e. transducers, acoustic releases, canister, etc.) is $20 \mathrm{~kg}$ and $6 \mathrm{~kg}$ in air and water respectively. The mass of the entire transmit mooring minus the concrete anchors is $46 \mathrm{~kg}$ and $19 \mathrm{~kg}$ in air and water respectively. The mooring lengths for both receive and transmit configurations are roughly six meters. 


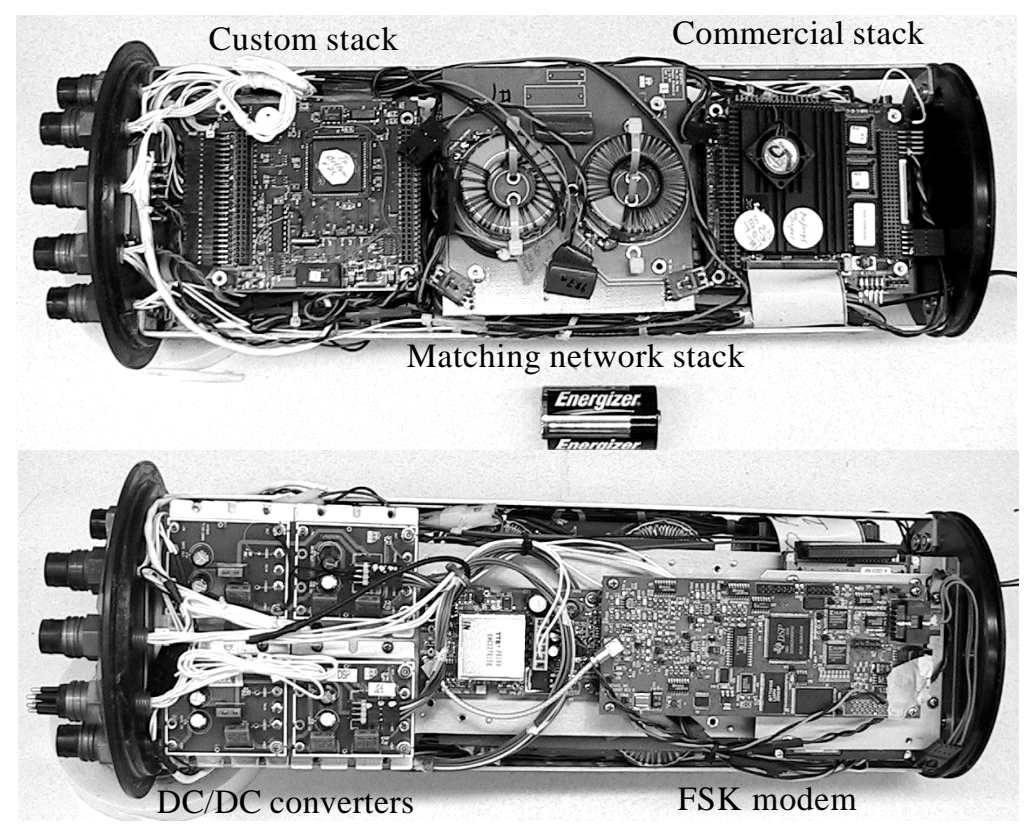

FIGURE 2. Top and bottom view of the electronics within the Telesonar Testbed. A D-cell battery is shown for scale.

\section{Electrical Description}

The electronics within the canister can be divided up into six main subsystems: 1 . custom circuit card stack, 2. matching-network stack, 3. commercial circuit card stack, 4. DC/DC converters, 5. amplifier, and 6. FSK modem (Figures 2, and 3).

The custom stack is comprised of four cards sandwiched via two 50-pin connectors on each board. The master controller (MC) board coordinates mission execution; the power-distribution board (PDB) routes power to the subsystems under MC control; the $\mathrm{RF}$ board allows control of the instrument via RF link when the instrument is sealed but not deployed; and finally, the amplifier board sets the transmitter gain and routes the signal for transmission to the appropriate matching network/transducer combination.

A matching network is required for each of the three transmit bands. The matching networks are not simply transformers for boosting signals fed to the transducers and canceling the capacitive reactance of the transducer, but rather they are Norton transformations made up of two or more toroidal inductors and several highvoltage/current capacitors providing favorable load characteristics to the amplifier and equalizing the overall response for a wider useable transmit bandwidth, Fig 4.

Three PC-104 compliant boards comprise the commercial board stack: single-board computer (SBC), 12-bit A/D, and a 12-bit D/A board. The SBC coordinates the sourcing of hard-disk-drive stored data to the D/A for transmission. It also coordinates the digitizing of received waveforms for storage to the hard-disk drive. 


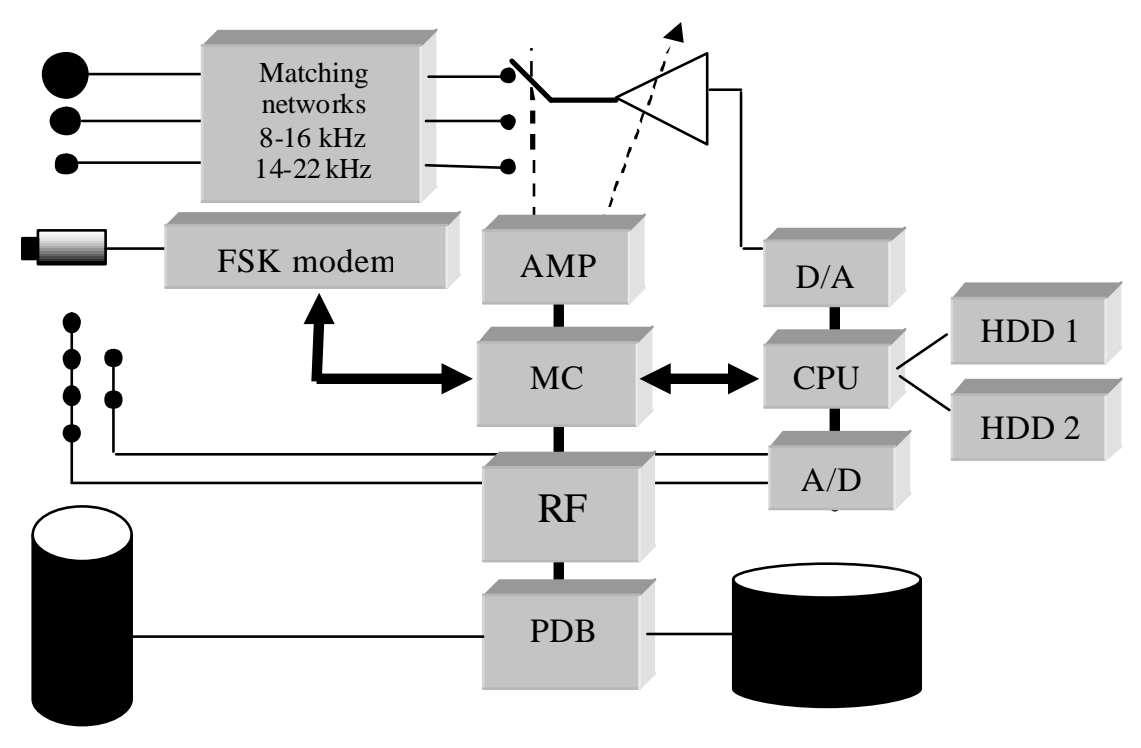

FIGURE 3. Block diagram of the Telesonar Testbed.

Finally, the SBC logs all activity and error messages to file for post-mortem missionexecution analysis.

There are four Vicor Inc. DC/DC converter modules that provide \pm 48 volts to the amplifier, 5 volts to the custom and commercial circuit card stacks, and 28 volts to the FSK modem.

The 200-watt, class A/B amplifier by APEX is mounted to the endcap for heat dissipation.

The commercial FSK modem by Benthos Inc. is used for remote control and status once the instrument is deployed.

\section{Instrument Reliability}

Reliability is crucial for autonomous, ocean-deployed systems in which experimental delays are expensive. There are several features of the Testbed instrument that ensure successful mission execution. The mission coordinator is an 8bit microcontroller (PIC17C756A) residing on the MC board. The simplicity of the hardware architecture and the C-code that runs on it, virtually eliminates unexpected code execution. A GNU general public licensed Real-Time Operating System (RTOS) TICS is required to allow the instrument to simultaneously execute multiple tasks. This simple, yet powerful RTOS which is run under DOS (Disk Operating System) on the SBC, provides a robust, reliable software platform for carrying out mission commands from the MC board issued over a serial RS-232 connection between them. This serial link is also used by the MC to verify correct command execution. The MC reboots the SBC if it suspects it has "hung" or is improperly executing commands. As a last resort, the FSK modem can be used to remotely toggle a hardware line that resets the microcontroller on the MC board if topside scientists believe the microcontroller is not functioning properly. 

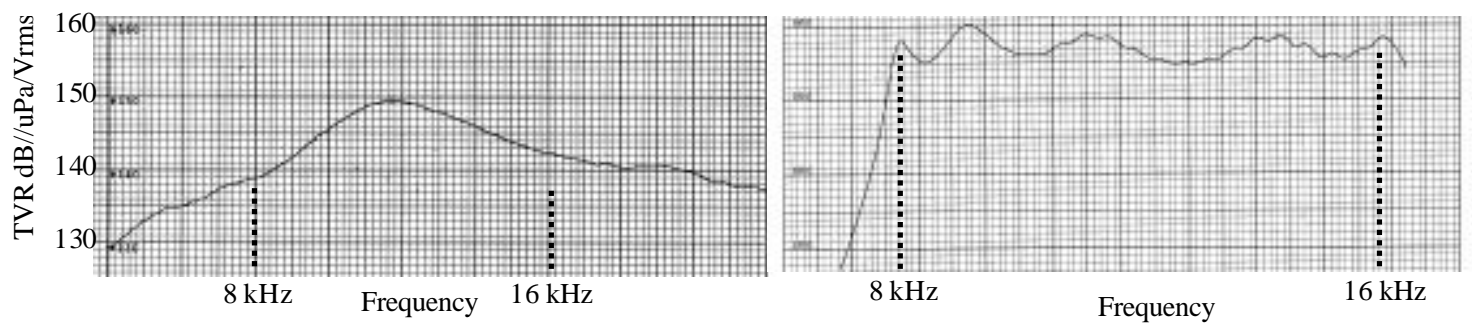

FIGURE 4. Transmit voltage response of the ITC-1007 (International Transducer Corp.) uncompensated (left), with Norton transformation matching network, (right).

\section{Salient Features}

The testbed instrument is capable of sourcing arbitrary waveforms at a maximum of $183 d B$ re $1 \mu$ Pascal @ 1 meter, continuously, in the three frequency bands mentioned above. A single external transmit battery pack provides 24 hours of transmit time at the maximum source level. Strapping on another similar-sized battery canister doubles the transmit time to 48 hours. The FSK modem link allows for command submission and is also used to monitor system status including disk-space usage, proper mission execution, and battery voltages. Long-term experiments for studying seasonal variations in acoustic signal propagation and communication performance is made possible by a low-power microcontroller and the sleep mode of the FSK modem. These are the only two devices that are continuously powered. Lastly, ground work has been laid for adding realtime modem development on a general-purpose Texas Instruments' 32-bit floating-point DSP processor.

\section{DATA ANALYSIS}

As mentioned above, the Telesonar Testbeds have been used for a variety of experiments to address different issues in underwater acoustic communications. Here we discuss one particular application in which we studied various techniques for carrier estimation and symbol synchronization. In particular, the next few sections apply six carrier estimation and six symbol synchronization techniques to QPSK (quadrature phase shift keying) signals collected by receiver-configured Testbeds during the KauaiEx and ElbaEx Experiments.

The highly-distortive nature of the underwater medium [1] has governed the development of synchronization methods used there [2,3]. Typically, power and time is expended on a preamble for estimating Doppler, symbol synchronization, and equalizer initialization. Contemporary free-space RF systems cannot afford this luxury and thus synchronization techniques that rely on secondary properties of the communication signals have been developed. We were curious as to how well these techniques would perform against signals transmitted through the underwater channel. A complete description of the techniques described next can be found in a yet to be published thesis [4]. 


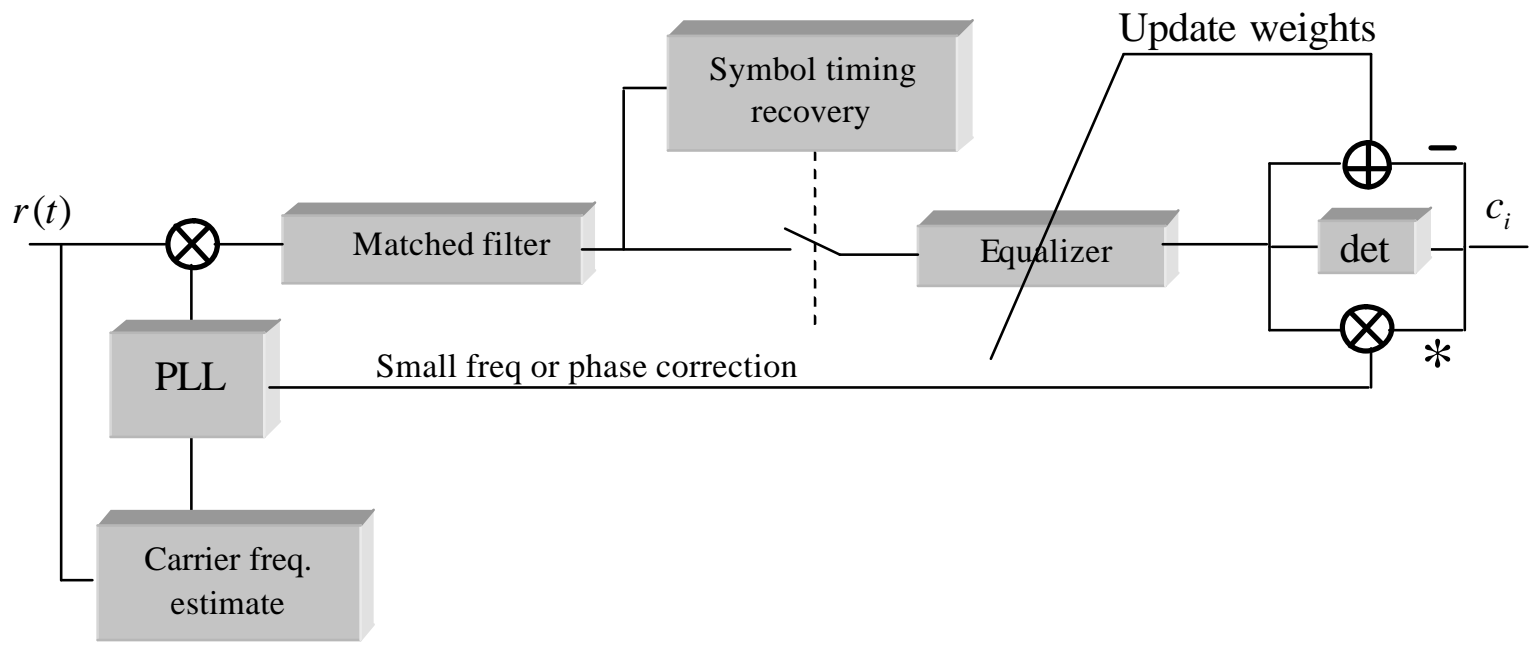

FIGURE 5. Simple receiver block diagram.

\section{Synchronization Basics}

In order to baseband a received passband signal for subsequent processing, receivers in phase-coherent systems are required to estimate the frequency and phase of the carrier. In older systems, these tasks were assisted by aids that were sent along with the modulated signal. These and other synchronization aids have generally been phased out due to disadvantages they each posses. These aides either require more bandwidth or power, or they decrease throughput, all precious commodities. Therefore, most receivers are expected to extract both carrier and symbol timing from the received modulated signal by exploiting secondary signal properties.

In most modems, large frequency offsets are taken care of before other synchronization processes are started. Therefore, methods operating on the passband signal directly without access to detected symbols must be used. Once the frequency of the carrier has been estimated and is within 0.1 of $\mathrm{T}$ (symbol period), a symboltiming loop can be started. Symbol timing estimation can be carried out in the presence of small carrier frequency error; implying that, obviously, phase-lock has not been achieved. Nevertheless, symbol-timing detector performance generally improves with decreased carrier frequency offset.

Once symbol sychronization has been achieved, symbols can then be delivered to a slicer, via an adaptive equalizer, that forms a maximum likelihood decision on the delivered symbol. Then, the angle between the delivered symbol and the intended location provide the carrier frequency estimator a phase-error signal for phase locking to the carrier, and provides the error signal for updating the equalizer weights. Figure 5 shows a simple high-level block diagram of a receiver.

The balance of this paper will now briefly cover the six carrier frequency estimators and six symbol-timing synchronizers. All six carrier frequency estimation methods discussed operate independent of symbol timing and vice versa. Furthermore, the symbol timing methods all are able to operate with some residual rotation of the in- 


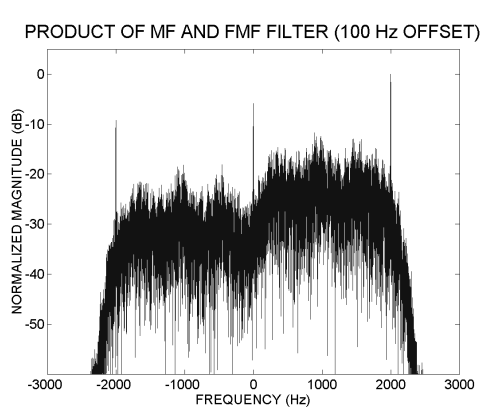

(a)

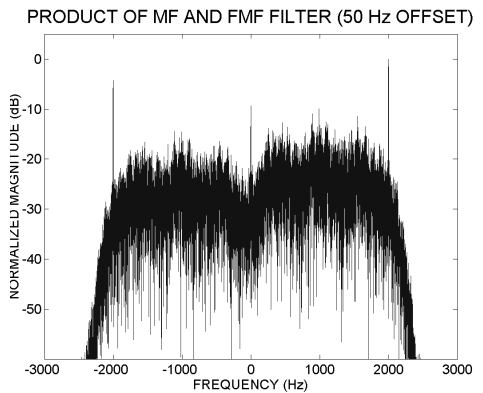

(b)

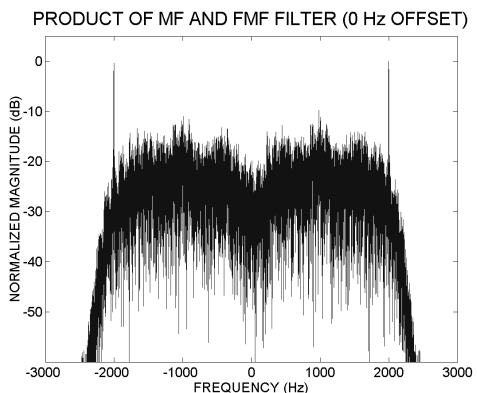

(c)

FIGURE 6. Product of the matched filter and frequency matched filter. Plot (a) and (b) plots show a DC line proportional to a 100 and $50 \mathrm{~Hz}$ offset between the up- and down-convert frequencies. Notice that the DC line is absent when the correct down-convert frequency is used (plot (c)).

phase and quadrature symbols. Lastly, because phase-locked loops (PLLs) are at the heart of most synchronization systems, at least one, second-order proportional-plusintegral PLL [4] was integrated into each synchronization technique's closed-loop implementation. Lastly, there is little latitude in the open-loop implementation of the methods; however, the closed-loop solutions leave more room for implementation flexibility and thus may bias the performance comparisons. Nevertheless, every effort was made to put the techniques on common footing.

\section{Carrier Frequency Estimation Introduction}

In QPSK communication systems, the information is carried by the phase modulation of a carrier. It is therefore essential to replicate exactly the frequency and phase of the carrier so that the in-phase and quadrature projections that were impressed upon the carrier can be determined. Franks in [5] offers a nice tutorial on symbol and carrier synchronization. With few exceptions, synchronizers for frequency-selective fading channels are adopted from methods using the AWGN (additive white Gaussian noise) noise models; not because of their superior performance but rather because of a shortage of more optimal solutions. Most carrierfrequency estimators for the AWGN channel rely upon the spectral symmetry of the received signal to provide a center-of-gravity estimate of the carrier frequency. The underwater channel's frequency-selective fading, frequency dependent absorption, and the transmit and receive instrument's non-flat transmit and receive response respectively, all couple together to present a non-symetric spectrum that biases centerof-gravity techniques.

\section{Technique Descriptions for Carrier Frequency Estimation}

The six techniques for carrier frequency estimation can be further segregated into the following broad categories: 1. maximum likelihood, 2. center-of-gravity, 3. spectral line.

The maximum likelihood frequency estimator forms the product of two filters, the matched filter and the frequency matched filter (MF-FMF) [4, 6]. The DC term of this product is proportional to the frequency offset between the up- and down-convert 


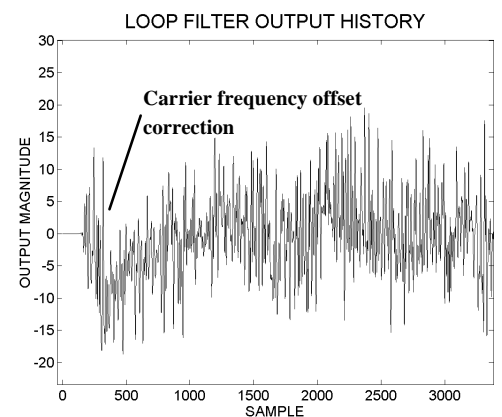

(a)

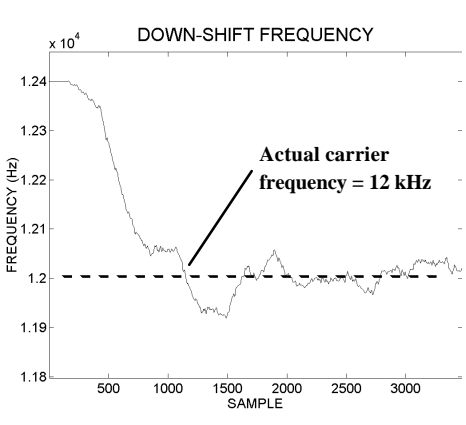

(b)

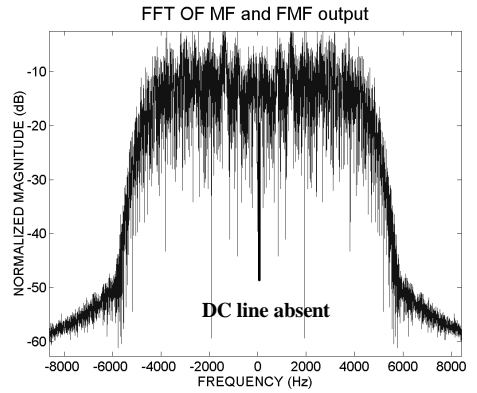

(c)

FIGURE 7. Closed loop implementation of the matched filter / frequency matched filter

frequencies. Figure 6 shows the output of these two filters as a function of the residual frequency offset. Figure 7 depicts the dynamic operation of the closed-loop solution.

The conjugate product estimator directly estimates the error in the down-convert or basebanding frequency. The underlying idea is rather simple. Suppose that the initial down-convert frequency is in error. If the basebanded signal is oversampled, the correlation from sample-to-sample will be a function of the sinusoidal modulation of the in-phase and quadrature data caused by this frequency mismatch. To find the rotation rate and thus the frequency error, the angle between successive samples is determined. The modulation contributes to the noise in this estimate and therefore, the conjugate product is fed to an averager before taking the arctan.

Another non-data-aided frequency estimator uses a bank of frequency-translated matched filters. The idea here is again simple. The received passband signal is fed to a bank of frequency-shifted matched filters that span the expected range of possible frequency shifts due to Doppler or differences in transmitter and receiver clocks. The frequency spacing of each match filter is dictated by the amount of residual frequency offset a down-stream carrier PLL can tolerate. In other words, this maximumsearching estimator provides an estimate of the carrier frequency but not the phase. The magnitude squared of each matched filter output is then summed over $L$ symbols and is fed to a peak detector. If the symbols are highly oversampled, then this technique provides both the frequency offset and symbol timing as depicted by the ambiguity surface in Fig 8.

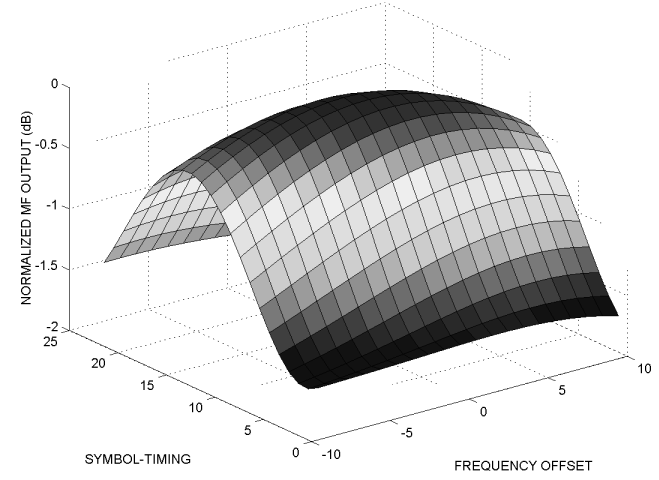

FIGURE 8. Ambiguity surface created by frequency-translated matched filter. Symbol timing and down-convert frequency can be gleaned from plot. 


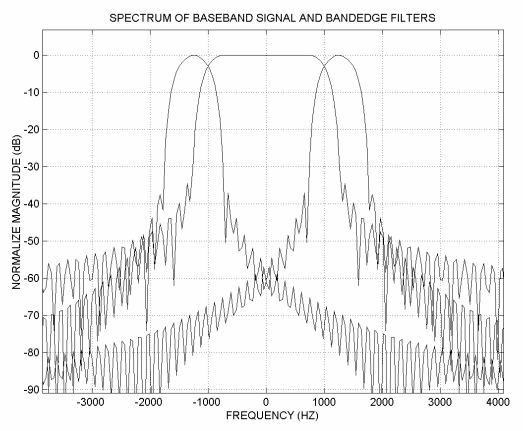

FIGURE 9. Spectrum of band-edge filters plotted on top of signal spectrum

The FFT-window technique is a center-of-gravity method that compares spectral energy in the signaling band for a collection of down-convert frequencies. The carrier frequency associated with the maximum power in the signaling band is then chosen as a first-order approximation of the down-convert frequency. Subsequent processing will eliminate any residual frequency offset and will phase-lock the local generated carrier to incoming passband signal.

Another center-of-gravity technique that relies upon the signaling-band symmetry, compares the energy in the right and left band edges. The generation of the band-edge filters is discussed in [4]. The filters are shown in Fig 9. These so-called band-edge filters are created by the frequency-domain product of the matched filter and the derivative matched filter. Two advantages of this technique are its rapid estimation of the frequency offset, and its operation without a cross product of any kind. Unfortunately, it will provide a biased estimate if the channel distorts the signal leaving unequal energy in the two band edges.

The spectral-line method is the only technique that operates off of the carrier directly. The modulation of a QPSK signal can be stripped by sending it through a $4^{\text {th }}$ power non-linearity. This method can provide both the frequency and phase of the carrier for sufficient receive signal-to-noise (SNR). At reduced SNRs this method provides the best estimate of the carrier frequency if the output of the PLL is averaged over many symbols. Figure 10 shows the PLL cycle slipping as it attempts to lock

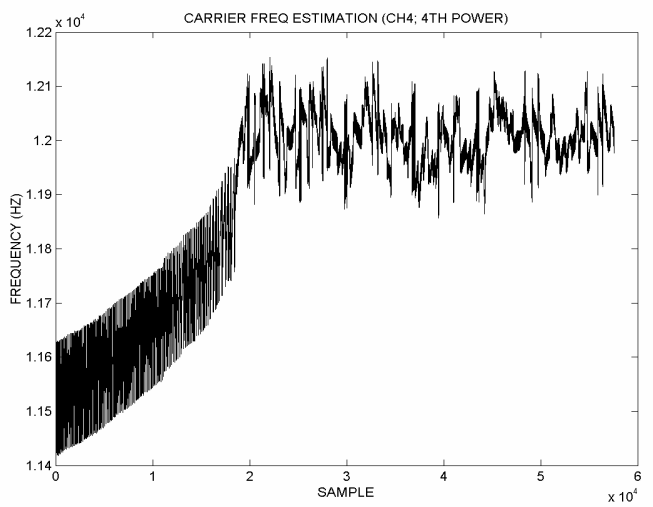

FIGURE 10. Fourth-power carrier frequency estimation. Notice non-linear cycle slipping before loop tracks close to the actual carrier frequency of $12 \mathrm{kHz}$. 


\begin{tabular}{|l|c|c|c|}
\hline \multicolumn{3}{|l|}{ TABLE 1. Carrier estimation techniques } & \\
\hline \multicolumn{1}{|c|}{ Technique } & North Elba & South Elba & HFX \\
\hline 4th power & +3 & -10 & +7 \\
\hline Frequency offset MF & +40 & -120 & -240 \\
\hline FFT window & +40 & -120 & -240 \\
\hline Band-edge filters & +40 & -120 & -240 \\
\hline Conjugate product & -20 & -245 & -221 \\
\hline FMF-MF & -40 & -240 & -300 \\
\hline
\end{tabular}

onto the carrier of $12 \mathrm{kHz}$. Once the PLL leaves a non-linear operating region (i.e. after cycle slipping), it tracks frequencies at or nearby $12 \mathrm{kHz}$.

Table 1 shows the results of the carrier frequency estimation techniques applied to data collected by the Telesonar Testbed during the KauaiEx and ElbaEx Experiments. As described above, the non-flat received signal spectrum significantly biases the result of all techniques except the $4^{\text {th }}$-power method. Since the $4^{\text {th }}$ power technique is the only one that operates off of the carrier directly, it is insensitive to a non-flat receive spectrum and performs well. The actual carrier frequency was $12 \mathrm{kHz}$, the symbol rate was $2 \mathrm{~K}$ symbols/second, and the sample rate was $48 \mathrm{ksps}$.

\section{Symbol Timing Estimation}

The function of any symbol timing synchronizer is to maximize the SNR of the symbol delivered to down stream signal processing blocks in a receiver. Typically the received matched filter is a copy of the pulse shape that was sent. Therefore, the matched filtering operation on the received data is a correlation process that produces peaks when the received pulse is time aligned with the matched filter. These peaks represent the symbol estimate with the maximum SNR. Therefore, the timing recovery algorithm must provide sampling instants with the correct frequency and phase for sampling these peaks. If Nyquist pulses are used (e.g. raised cosine) then in an ideal channel exhibiting only a time shift, sampling at the peak will provide the best, intersymbol-interference free, estimate of the symbol. Tutorials on symbol timing are rare but can be found in [5, 7-9].

There are two main classifications of techniques for estimating symbol timing: Decision Directed (DD) or Data Aided (DA), and non-decision directed (NDD). This paper will deal only with NDD methods or those that estimate sampling instants without the aid of detected symbols. The following few paragraphs will describe the 6 techniques applied to data distorted by underwater channels.

The function of a timing recovery algorithm boils down to estimating $\tau$. This parameter accounts for the time lag between transmitter and receiver, and is unknown but non-random, and therefore Maximum Likelihood (ML) techniques direct us toward an optimal timing recovery algorithm. An approximation to the ML algorithm is the so called matched filter/derivative matched filter (MF-DMF) technique [10]. This method can be justified heuristically by observing that it will attempt to move the sampling phase until the derivative of the baseband signal is zero, which occurs at the peaks of the signal. 


\begin{tabular}{|l|c|c|c|}
\hline \multicolumn{5}{|c|}{ TABLE 2. Performance of symbol timing techniques. } \\
\hline \multicolumn{1}{|c|}{ Technique } & North Elba & South Elba & HFX \\
\hline Gardner & 0.036 & 0.11 & 0.05 \\
\hline MF-DMF & 0.28 & 1.3 & 1.9 \\
\hline MF-FMF & 0.46 & 0.36 & 1.6 \\
\hline Early-late Gate & 0.75 & 2.5 & 1.9 \\
\hline Square with BE filter & 0.53 & 2.6 & 1.9 \\
\hline Square without BE filter & 5.6 & 6.2 & 5 \\
\hline
\end{tabular}

The early-late gate symbol synchronizer [11] is a ML approximation that exploits the symmetry properties of the matched filter output. This technique requires three samples and the timing is adjusted so that the first and the third samples are equal to one another. That way, the middle sample should be at or close to the peak.

Another very popular symbol timing estimator was presented by F. M. Gardner in [12] and is a minimum-likelihood method since it operates on the zero-crossings instead of the peaks of the basebanded signal. This algorithm adjusts the timing so that the sample between the symbols is zero. As Table 2 shows, this algorithm works surprisingly well, outperforming the ML techniques.

The MF-FMF described above not only produces a DC line proportional to the error in the down-convert frequency, but also produces spectral lines at the symbol rate. A PLL can then be locked to this line and the sampling instants generated by picking the peaks of the PLL output.

Passing a QPSK signal through a squaring non-linearity produces a spectral line at the symbol rate. These lines are created by the convolution in frequency of the spectrum of the received signal with a copy of this spectrum. Remember that the product in time is equivalent to convolution in frequency. Therefore, when the spectrum perfectly overlaps a DC term forms. Also, when the band edges overlap a spectral component at the symbol rate will form.

Since the symbol timing information is contained in the band edges, the squaring algorithm can be improved if we reject the signaling band through the use of a bandedge filter. Table 2 demonstrates the improved performance of this modification to the squaring algorithm.

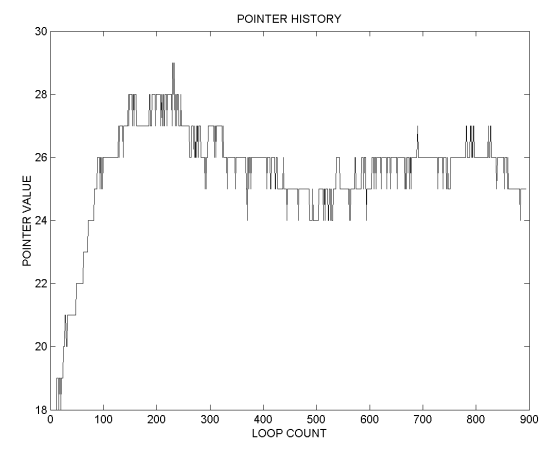

FIGURE 11. Dynamic operation of the Gardner zero-crossing method 
Each of the six techniques just described were implemented in a closed-loop form using a second-order loop filter. The performance metric was the variance of the pointer value that the loop produced. Since there are 24 samples available per symbol, these tracking loops converged to one of 24 possible pointer values. Figure 11 shows the dynamic operation of the Gardner loop. Table 2 ranks the performance of the six techniques tested.

\section{CONCLUSIONS}

It is clear from Table 1 that all of the carrier frequency estimation techniques except for the $4^{\text {th }}$-power method, perform poorly when working on signals that have been distorted by an underwater channel. On the other hand, Table 2 indicates that the NDD symbol timing techniques appear to be adequate for estimating symbol locations.

The Telesonar Testbed will continue to be utilized for underwater communication and high-frequency acoustic propagation research

\section{ACKNOWLEDGMENTS}

The KauaiEx Group is: Michael B. Porter, Paul Hursky, Martin Siderius (SAIC), Mohsen Badiey (Univ. Delaware), Jerald Caruthers (Univ. Southern Miss.), Daniel Rouseff, Warren Fox (Univ. Washington), Chritian de Moustier, Brian Calder, Barbara J. Kraft (Univ. New Hampshire), Keyko McDonald (SPAWARSYSCEN), Peter Stein, James K. Lewis, and Subramaniam Rajan (Scientific Solutions).

We would like to extend our gratitude to the SACLANT Undersea Research Centre and all involved SACLANT scientists and engineers who made the Elba Experiment a success. A special thanks to the Chief Scientists Finn Jensen and Mark Stevenson.

\section{REFERENCES}

1. Rice, J. A., "Acoustic Signal Dispersion and Distortion by Shallow Undersea Transmission Channels," Proc. NATO SACLANT Undersea Research Centre Conf. on High-Freq. Acoustic in Shallow Water, Lerici, Italy, pp. 435-442, July 1997.

2. Stojanovic, M., Catipovic, J. A., and Proakis, J. G., "Adaptive multi-channel combining and equalization for under water acoustic channels," Journal of the Acoustical Society of America, vol. 94, pp. 1621-1631, 1993.

3. Stojanovic, M., Catipovic, J. A., and Proakis, J. G., "Phase-coherent Digital Communications for Underwater Acoustic Channels," IEEE Journal of Oceanic Engineering, vol. 19, 100-111, January 1994.

4. McDonald, V. K., Carrier and Symbol Synchronization Techniques for Phase-coherent Communication Applied to Shallow Water Ocean Channels, 2004 (yet to be published).

5. Franks, L. E., "Carrier and Bit Synchronization in Data Communication - A Tutorial" Review, IEEE Transactions on Communications, vol. Com 28, no. 8, August, 1990. 
6. Harris, F. J., "Band edge Filtering and Processing for Carrier and Timing Recovery," COMCON-7 Athens Greece, 28 June - 2 July 1999.

7. Bennett, W. R., "Statistics of Regenerative Data Transmission," BSTJ 37 pp. 1501-1542, Nov. 1958.

8. Gitlin R. D., and Hayes, J. F., "Timing Recovery and Scramblers in Data Transmission," BSTJ 54(3), march 1975.

9. Aaron, M. R., "PCM Transmission in the Exchange Plant," BSTJ 41 pp. 99-141, January 1962.

10. Saltzberg, B. R., "Timing Recovery for Synchronous Binary Transmission," BSTJ, pp. 593-622, March 1967.

11. Gitlin R. D., and Salz, J., "Timing Recovery in Pam Systems," BSTJ 50(5) p. 1645, May and June 1971.

12. Gardner, F. M., “A BPSK/QPSK Timing-Error Detector for Sampled Receivers," IEEE Transactions on Communications, Vol. COM-34, pp. 423-429, May 1986. 